\section{Effect of beinaglutide treatment on weight loss in Chinese patients with type 2 diabetes mellitus and overweight/obesity}

\author{
Guiying Wang ${ }^{1 *}$ \\ https://orcid.org/0000-0001-8072-0240 \\ Peng $\mathrm{Wu}^{2 *}$ \\ https://orcid.org/0000-0002-8986-0204 \\ Yan Qiu ${ }^{2}$ \\ https://orcid.org/0000-0002-4540-3183 \\ Xin Dong ${ }^{2}$ \\ https://orcid.org/0000-0002-3876-1767 \\ Yingbin Wang ${ }^{1}$ \\ https://orcid. org/0000-0001-8575-065X \\ Yanjun $\mathrm{Chi}^{1}$ \\ https://orcid. org/0000-0003-3428-7225 \\ Fengjuan Zhang \\ https://orcid.org/0000-0003-4029-1482 \\ Yinyu $\mathrm{Li}^{1}$ \\ https://orcid.org/0000-0001-7808-7634 \\ Jimin Zhang ${ }^{1}$ \\ https://orcid.org/0000-0001-5357-5674 \\ Zhengli Huang' \\ https://orcid org/0000-0003-2044-2802 \\ Xifeng $\mathrm{Du}^{2}$ \\ https://orcid. org/0000-0003-0070-8677 \\ Zhiqiang $\mathrm{Du}^{2}$ \\ https://orcid.org/0000-0003-0686-1891
}

\begin{abstract}
Objective: To evaluate the effect of beinaglutide on weight loss and plasma protein patterns of inflammation/obesity relevant cytokines and biomarkers. Materials and methods: This study involved 36 adult patients with a body mass index (BMI) of $\geq 24 \mathrm{~kg} / \mathrm{m}^{2}$ and T2DM. Beinaglutide was administered for three months. Changes in body weight, fasting plasma glucose (FPG) level, $2 \mathrm{~h}$ postprandial plasma glucose (2h-PG) level, glycosylated hemoglobin ( $\mathrm{HbA1c}$ ) level, BMI and visceral and subcutaneous fat areas were measured at baseline and after three months of treatment. In addition, relevant inflammation/obesity cytokines and biomarkers were measured. Results: After three months, beinaglutide treatment led to significant changes, including in body weight, BMI, FPG level, HbA1c level, visceral and subcutaneous fat areas. In addition, serpin E1, leptin, C-reaction protein (CRP) and tumor necrosis factor- $\alpha$ (TNF- $\alpha$ ) also decreased significantly. The plasma protein concentrations of CRP (Log2 transformed) were found to be positively correlated with the percentage of weight loss $(R=0.514$ and $p$-value $=0.021)$. Conclusion: Beinaglutide treatment resulted in weight loss, plasma glucose control and anti-inflammatory effects in patients with T2DM and overweight/ obesity. Arch Endocrinol Metab. 2021;65(4):421-7
\end{abstract}

\section{Keywords}

T2DM; overweight; obesity; GLP-1
1 Department of Endocrinology and Metabolism, The First Affiliated Hospital of Datong University, Datong, Shanxi, China ${ }^{2}$ Shanghai Benemae Pharmaceutical Corporation, Shanghai, China

${ }^{*}$ Co-first Author
Correspondence to:

Zhiqiang Du

Shanghai Benemae Pharmaceutical Corporation

916 Ziping Road, 201321, Shanghai, China

duzhiqiang@benemae.com

Received on June/21/2020 Accepted on Apr/29/2021

DOI: $10.20945 / 2359-3997000000388$

\section{INTRODUCTION}

$\mathrm{T}$ The number of people with overweight/obesity has been steadily increasing. Obesity has been recognized as one of the greatest public health concerns worldwide, especially in developed countries (1). A study conducted in different parts of China revealed that the prevalence of obesity is increasing (2). Obesity 
increases the morbidity of several chronic diseases and also increases the mortality of cardiovascular diseases, diabetes, cancers and musculoskeletal disorders (3-7). Among Chinese patients (aged 35-74 years) with type 2 diabetes mellitus (T2DM) in $2010,28.3 \%$ and $10.1 \%$ of males and $31.3 \%$ and $16.8 \%$ of females were considered overweight and obese, respectively. It is reported that approximately 3.32 million T2DM events were attributable to overweight and obesity (8).

Several GLP-1 receptor agonists (GLP-1RA) have been promoted for the treatment of T2DM and have multiple potential effects on hyperglycaemia, cardiovascular and liver disease $(9,10)$. Additionally, the mechanism by which GLP-1RA treatment leads to weight loss has been reported (11). Among these GLP-1RAs drugs, beinaglutide is the only prandial GLP-1 (7-36) receptor agonist with a $100 \%$ protein sequence identity to human 7-36 GLP-1. A retrospective observational real-world study reported that beinaglutide significantly reduced the bodyweight of the patients with T2DM and obesity while lowering the plasma glucose and glycosylated hemoglobin (HbAlc) levels (12).

However, inflammation/obesity relevant cytokines and biomarkers from plasma in patients with T2DM treated with beinaglutide are still unknown. Our study aimed to evaluate the effect of beinaglutide on weight loss and plasma protein patterns of inflammation/ obesity relevant cytokines and biomarkers.

\section{MATERIALS AND METHODS}

\section{Study design and patients}

This study was a single-centre, prospective, openlabel, self-controlled real-world study (RWS) involving 36 patients with overweight/obesity and T2DM. Patients were recruited from the First Affiliated Hospital of Shanxi Datong University. All patients gave their informed consent before receiving beinaglutide treatment. This study was approved by the Ethics Committee of the First Affiliated Hospital of Shanxi Datong University in China and conducted in accordance with the ethical guidelines of the Declaration of Helsinki (1975).

The inclusion criteria were adult patients $(\geq 18$ years old) with a body mass index (BMI) of $\geq 24 \mathrm{~kg} / \mathrm{m}^{2}$ and a diagnosis of T2DM according to the WHO 1999 criteria. The exclusion criteria were patients with clinically significant cardiac, central nervous system, rheumatic or cancer diseases, females who were pregnant or planning to become pregnant, patients enrolled in another clinical trial within the past three months, patients who used a GLP-1 receptor agonist or weight loss drug in the past three months, patients with long-term use of glucocorticoids that led to being overweight/obese, acute diabetic complications, infections or other endocrine diseases, patients with a history of pancreatitis, cancer of the pancreas, type 2 multiple endocrine neoplasia syndrome, or medullary thyroid carcinoma, or patients with inflammatory intestinal diseases or diabetic gastric paretitis.

Beinaglutide injections were administered twice per day before meals, with a starting dose of 0.06 mg per injection each week, climbing to $0.1 \mathrm{mg}$ per injection for three months. Bodyweight (BW), BMI, visceral fat area (VFA), subcutaneous fat area (SFA), waist circumference (WC), HbAlc level, fasting plasma glucose (FPG) level, 2 h postprandial plasma glucose (2h-PG) level, heart rate and blood pressure were measured at baseline and after three months. Besides, plasma was collected for cytokines and biomarkers detection before and after three months of treatment with beinaglutide. The primary end-point was weight loss after three months compared to the baseline. The secondary end-points were changes in FPG, $2 \mathrm{~h}$ PG, HbAlc, BMI, VFA and SFA after three months compared to the baseline.

\section{Cytokines and biomarkers measurement}

Plasma was separated within $24 \mathrm{~h}$ from the patients at baseline and three months later and then stored at $80^{\circ} \mathrm{C}$ for future analysis. The Human Obesity Premixed Mag Luminex Performance Assay Kit (FCSTM08-10, R\&D Systems) was used to analyse ten inflammation/ obesity related plasma cytokines and biomarkers including Adiponectin/Acrp30, C-Reactive Protein/ CRP, Complement Factor D/Adipsin, Serpin El/ PAI-1, CCL2/JE/MCP-1, IL-6, IL-10, Leptin/ $\mathrm{OB}$, resistin and TNF- $\alpha$ which sensitivities were $6.4 \mathrm{pg} / \mathrm{mL}, 1.4 \mathrm{pg} / \mathrm{mL}, 0.16 \mathrm{pg} / \mathrm{mL}, 1.8 \mathrm{pg} / \mathrm{mL}$, $0.36 \mathrm{pg} / \mathrm{mL}, 0.13 \mathrm{pg} / \mathrm{mL}, 7.69 \mathrm{pg} / \mathrm{mL}, 0.85 \mathrm{pg} / \mathrm{mL}$, $0.20 \mathrm{pg} / \mathrm{mL}$ and $0.60 \mathrm{pg} / \mathrm{mL}$, respectively. The panel is a Luminex system that uses a fluorescent bead system and $\mathrm{CV}$ (coefficients of variation) of the cytokines/ biomarkers are all below $20 \%$. All assay procedures were performed as described by the manufacturer. 


\section{Quantification of visceral and subcutaneous fat area}

Abdominal bioelectrical impedance analysis (BIA) (OMRON, HDS-2000 DUALSCAN) was used to estimate VFA and SFA (13). The VFA was calculated by calculating the total sectional area of the abdomen with the BIA between the umbilicus and the back.

\section{Statistical analyses}

Statistical analysis was conducted and boxplots were generated using $\mathrm{R}$ software (version 3.6.1). A normality test of the original data was performed using shapiro.test function using $\mathrm{R}$ software. The cytokines and biomarkers data were $\log 2$ transformed to check for normality. Baseline and treatment data from the clinical data set were analysed by paired t-tests based on the normality of the original data. Paired oneside Wilcoxon tests with an alternative hypothesis of "greater" were used for non-parametric tests of the non-parametric plasma cytokines and biomarkers data set. The p-value cut-off was set at 0.05 . Correlation analysis was performed using the cor.test function in $\mathrm{R}$ software using the Pearson method. Weight loss (weight change) percentage was equal to (baseline body weight $(\mathrm{kg})$-treatment body weight $(\mathrm{kg})) /$ baseline body weight $(\mathrm{kg})$.

\section{RESULTS}

\section{Baseline characteristics and clinical outcomes}

A total of 36 participants complied with the criteria for this study. Twenty-four males and twelve females, with an overall mean age of $46.25( \pm 13.55)$ years. Baseline characteristics and clinical outcomes after three months of treatment with beinaglutide are shown in Table 1 and Figure 1. In brief, bodyweight related physical indices such as BW, BMI, VFA, SFA, WC and hip circumference were reduced significantly after three months ( $\mathrm{p}$-value $<0.05)$. There were $63.9 \%(23 / 36)$ who lost $3 \%$ or more in weight, and $41.7 \%$ (15/36) lost more than 5\% (data not shown). HbAlc and FPG levels were also significantly reduced, while heart rate, systolic blood pressure and diastolic blood pressure did not. These results suggest that patients with T2DM and overweight/obesity could benefit from beinaglutide for weight loss and plasma glucose control.

\section{Inflammation/obesity related cytokines and biomarkers}

Plasma concentrations of the panel of ten cytokines and biomarkers were quantified using a Luminex detection platform. Changes compared to baseline and three months of treatment are shown in Table 2 and Figure 2.

Table 1. Summary of patient characteristics before and after treatment with beinaglutide

\begin{tabular}{|c|c|c|c|c|}
\hline & Number & Baseline & Treatment & p-value \\
\hline \multicolumn{5}{|l|}{ Demographics } \\
\hline Gender & 36 & $12 \mathrm{~F} / 24 \mathrm{M}$ & 12 F/24 M & NA \\
\hline Age (y) & 36 & $46.25( \pm 13.55)$ & $46.25( \pm 13.55)$ & NA \\
\hline \multicolumn{5}{|l|}{ Obesity index } \\
\hline Weight (kg) & 36 & $88.97( \pm 11.32)$ & $85.18( \pm 11.45)$ & $<0.001$ \\
\hline $\mathrm{BMI}\left(\mathrm{kg} / \mathrm{m}^{2}\right)$ & 36 & $31.63( \pm 3.55)$ & $30.61( \pm 3.32)$ & $<0.001$ \\
\hline HR (bpm) & 35 & $84.06( \pm 14.01)$ & $79.63( \pm 13.69)$ & 0.09115 \\
\hline $\mathrm{SBP}(\mathrm{mmHg})$ & 35 & $139( \pm 16.52)$ & $138.23( \pm 17.19)$ & 0.68123 \\
\hline $\mathrm{DBP}(\mathrm{mmHg})$ & 35 & $81.42( \pm 11.21)$ & $81.77( \pm 12.83)$ & 0.93020 \\
\hline Hip circumference (cm) & 36 & $108( \pm 7.02)$ & $104.68( \pm 7.16)$ & 0.00104 \\
\hline Waist circumference (cm) & 36 & $103.74( \pm 7.51)$ & $98.71( \pm 7.93)$ & $<0.001$ \\
\hline VFA $\left(\mathrm{cm}^{2}\right)$ & 36 & $158.81( \pm 23.11)$ & $120.6( \pm 38.79)$ & $<0.001$ \\
\hline SFA $\left(\mathrm{cm}^{2}\right)$ & 36 & $281.43( \pm 65.75)$ & $267.64( \pm 62.88)$ & 0.02626 \\
\hline \multicolumn{5}{|l|}{ Plasma Glucose test } \\
\hline $\mathrm{HbA1c}(\%)$ & 24 & $8.75( \pm 2.31)$ & $7.38( \pm 1.90)$ & $<0.001$ \\
\hline $\mathrm{FPG}(\mathrm{mmol} / \mathrm{L})$ & 20 & $10.01( \pm 3.96)$ & $8.62( \pm 3.87)$ & 0.008 \\
\hline 2h-PG (mmol/L) & 18 & $14.44( \pm 4.58)$ & $12.20( \pm 4.65)$ & 0.012 \\
\hline
\end{tabular}

F/M: female/male; BMl: body mass index; HR: heart rate; SBP: systolic blood pressure; DBP: diastolic blood pressure; VFA: visceral fat area; SFA: subcutaneous fat area; HbA1c, haemoglobin A1c; FPG: fasting plasma glucose; 2h-PG: 2-hour plasma glucose. Data are shown as Mean $\pm \mathrm{SD}$. 

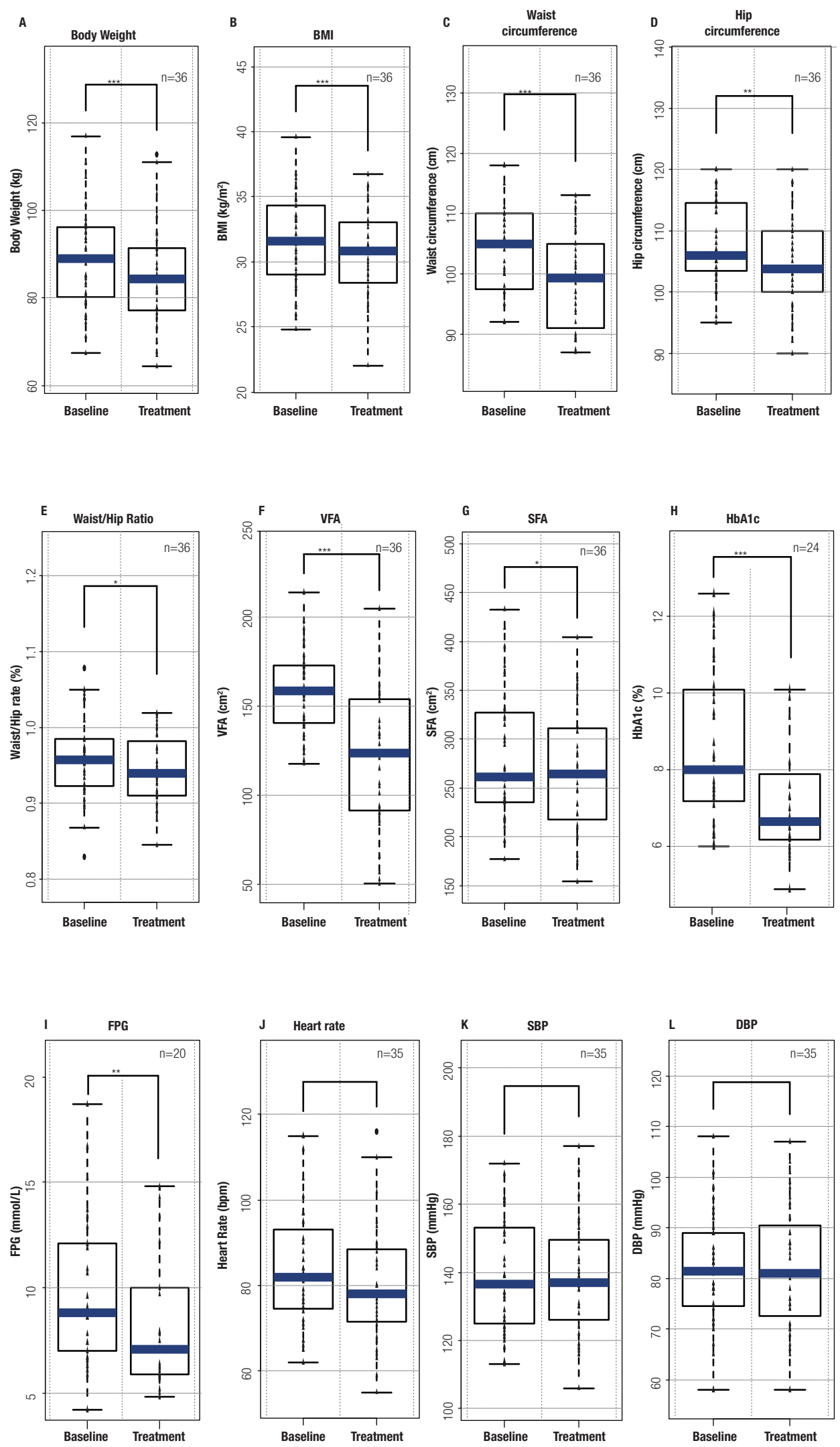

Figure 1. Comparison of baseline and treatment of patients receiving beinaglutide in body weight (A), body mass index (B), waist circumference (C), hip circumference (D), waist/hip rate $(E)$, visceral fat area $(F)$, subcutaneous fat area $(G), H b A 1 \mathrm{C}(H)$, fasting plasma glucose $(I)$, heart rate $(\mathrm{J})$, systolic blood pressure $(\mathrm{K})$ and diastolic blood pressure $(\mathrm{L}){ }^{*} \mathrm{p}<0.05 ;{ }^{* \star} \mathrm{p}<0.01 ;{ }^{* \star *} \mathrm{p}<0.001$. 
Serpin El, TNF- $\alpha$, Leptin and CRP were significantly decreased after treatment compared with baseline levels (p-value $<0.05$; Figure 2). The other five cytokines, adiponectin, CCL2, complement factor D, IL-10 and resistin, were not reduced, while IL-6 failed to be detected due to an extremely low signal. Correlation analysis showed that the $\log 2$ transformed plasma protein concentrations of CRP was positively correlated with the percentage of weight loss $(\mathrm{R}=0.514$, $\mathrm{p}$-value $=0.021$, Table 3 ).

\section{DISCUSSION}

Beinaglutide was approved as a novel drug for diabetes treatment and the clinical benefit of weight loss and plasma glucose control has also been reported (12). However, the protein pattern of plasma cytokines and biomarkers are still unclear. This study found a mean weight loss of $3.8 \mathrm{~kg}$ and $4.3 \%$ mean weight loss percentage after three months of treatment. Moreover, BMI, WC, hip circumference, visceral fat, and subcutaneous fat were also significantly decreased

Table 2. Changes of inflammation/obesity related plasma cytokines and biomarkers before and after treatment with beinaglutide

\begin{tabular}{lccc} 
& Baseline & Treatment & p-value \\
\hline Adiponectin $(\mathrm{ng} / \mathrm{mL})$ & $6046.23( \pm 4093.7)$ & $6739.87( \pm 4602.93)$ & 0.938 \\
CCL2 $(\mathrm{pg} / \mathrm{mL})$ & $121.84( \pm 49.87)$ & $105.81( \pm 29.64)$ & 0.084 \\
CRP $(\mathrm{ng} / \mathrm{mL})$ & $7627.52( \pm 8437.05)$ & $4197.02( \pm 6811.5)$ & $\mathbf{0 . 0 4 4}$ \\
Complement Factor D $(\mathrm{ng} / \mathrm{mL})$ & $6191.26( \pm 1483.5)$ & $6531.85( \pm 1774.22)$ & 0.554 \\
IL-10 $(\mathrm{pg} / \mathrm{mL})$ & $2.07( \pm 0.25)$ & $2.05( \pm 0.25)$ & 0.462 \\
Leptin $(\mathrm{ng} / \mathrm{mL})$ & $23.15( \pm 16.76)$ & $18.45( \pm 12.63)$ & $\mathbf{0 . 0 0 3}$ \\
Resistin $(\mathrm{pg} / \mathrm{mL})$ & $8.48( \pm 3.52)$ & $9.24( \pm 4.09)$ & 0.862 \\
Serpin E1 $(\mathrm{ng} / \mathrm{mL})$ & $48.02( \pm 24.2)$ & $25.1( \pm 13.15)$ & $\mathbf{0 . 0 0 0}$ \\
TNF- $\alpha$ (pg/mL) & $4.08( \pm 3.05)$ & $3.26( \pm 2.55)$ & $\mathbf{0 . 0 0 5}$ \\
IL-6 & NA & NA & NA \\
\hline
\end{tabular}

NA: no available data.
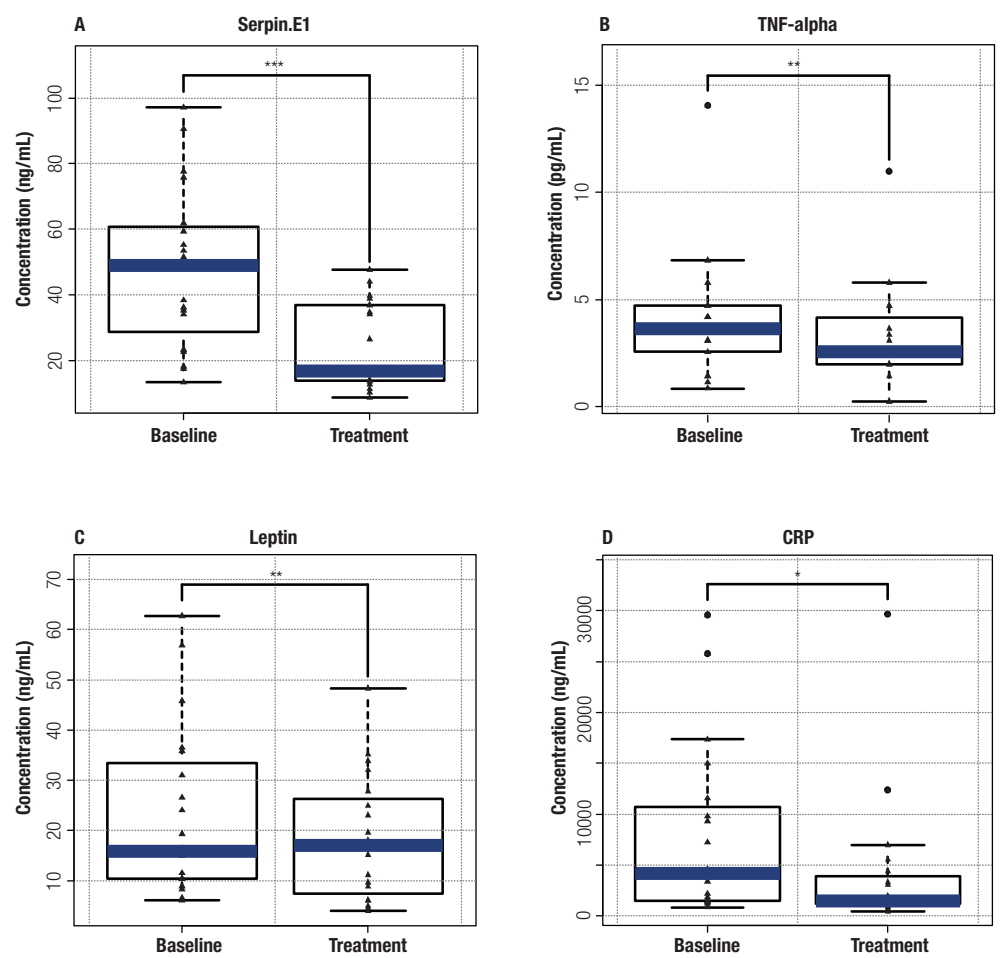

Figure 2. Comparison of baseline and treatment of patients receiving beinaglutide in 4 inflammation/obesity related cytokines/biomarkers. (A) Serpin E1, (B) TNF- $\alpha$, (C) Leptin, (D) C-reactive protein. ${ }^{\star} p<0.05$; ${ }^{\star \star} p<0.01$; ${ }^{\star \star \star} p<0.001$. 
Table 3. Correlation analysis between inflammation/obesity related plasma cytokines/biomarkers and weight loss percentage

\begin{tabular}{lcccc}
\hline Cytokines/biomarkers & $\mathbf{R}$ & p-value & R-log2 & p-value_log2 \\
\hline CRP & 0.398 & 0.082 & $\mathbf{0 . 5 1 4}$ & $\mathbf{0 . 0 2 1}$ \\
Adiponectin & -0.131 & 0.583 & -0.153 & 0.518 \\
TNF- $\alpha$ & -0.042 & 0.871 & -0.044 & 0.868 \\
Adipsin & 0.109 & 0.724 & 0.101 & 0.742 \\
IL-10 & 0.061 & 0.799 & 0.059 & 0.806 \\
CCL2 & -0.025 & 0.918 & -0.032 & 0.894 \\
Resistin & $\mathbf{0 . 5 4 6}$ & $\mathbf{0 . 0 1 3}$ & 0.051 \\
Serpin E1 & 0.033 & 0.89 & -0.008 & 0.973 \\
Leptin & 0.176 & 0.457 & 0.196 & 0.407 \\
IL-6 & NA & NA & NA & NA \\
\hline
\end{tabular}

$\mathrm{R}$ (and p-value): correlation (and p-value) between weight loss percentage and plasma cytokines/biomarkers concentrations; R-log2 (and p-value_log2): correlation (and p-value) between weight loss percentage and log2 transformed cytokines/biomarkers concentration; NA: no available data.

after beinaglutide treatment but not heart rate, diastolic blood pressure or systolic blood pressure (SBP). In addition, HbAlc, FPG and 2h-PG levels were also decreased. It has been reported that heart rate and SBP are affected by other GLP-1RA drugs with a long half-life and continuously activation of GLP-1R $(14,15)$. Therefore, the results of this study indicate the advantages of beinaglutide as a GLP-1 homolog with $100 \%$ protein sequence identity to human natural GLP1 and short half-life $(30 \mathrm{~min})(16,17)$.

Abdominal visceral fat and subcutaneous fat are independent risk factors for cardiovascular diseases and metabolic syndrome $(18,19)$. In this study, visceral fat decreased significantly by approximately $24 \%$ on average, while subcutaneous fat decreased by about $4 \%$ on average after three months of beinaglutide treatment. This finding suggests that beinaglutide primarily reduced body weight through visceral fat, which could be critical for patients with T2DM to reduce the risk of the related diseases.

In addition, we tested the exploratory cytokines and biomarkers of inflammatory or obesity to determine the related factors associated with weight loss after beinaglutide treatment. Serpin El is highly expressed in the plasma of patients with obesity and diabetes, indicating a link between them (20). Our data showed that serpin El was significantly downregulated in the plasma of the patients with T2DM and overweight/ obesity after three months of treatment with beinaglutide. This finding suggests that the downstream signalling pathway of the GLP-1 receptor might be associated with the regulation of Serpin El $(21,22)$.
Obesity is considered a chronic inflammatory disease. Macrophage migration in patients with obesity infiltrates into the vicinity of fat cells, leading to the secretion of inflammatory cytokines, thereby upregulating the expression of TNF- $\alpha$ and resistin (23). In this study, TNF- $\alpha$ was significantly reduced, suggesting the anti-inflammatory effect of beinaglutide. A parallel study has reported that inflammatory factors such as TNF- $\alpha$ decreased after GLP- 1 analog treatment in patients with diabetes and obesity $(24,25)$.

It has been reported that leptin decreased after treatment with diet control or GLP-1 drugs in patients with T2DM with effective or ineffective weight loss $(25,26)$. The serum leptin level in patients with obesity was higher than that in the normal weight group, and a decrease in body weight led to a decrease in leptin levels $(27,28)$. The results in our study are consistent with these studies, thus illustrating the importance of leptin in weight loss.

CRP is a widely investigated biomarker of inflammation in the pathogenesis of several chronic diseases such as cardiovascular disease and diabetes. It has been reported that a decrease in CRP is associated with direct weight loss (29). Mazidi's study showed that the level of CRP significantly decreased after treatment with GLP-1 RAs (30). The results of our study verified this finding.

Our research has several limitations. The limited sample size was an inevitable problem in our study, and the study would be informative if there were more data. However, these findings provide a foundation for future clinical research. Next, we will conduct a further study with a large population to validate our findings.

In summary, this study is the first to report the plasma protein pattern of inflammation/obesity related 
cytokines and biomarkers in Chinese patients with T2DM and overweight/obesity who were treated with beinaglutide. Beinaglutide also decreased bodyweight, as well as the plasma glucose and inflammatory levels. Furthermore, baseline level of CRP is related to the weight loss percentage of beinaglutide treatment in patients with T2DM and overweight/obesity.

Authorship: G.W. and Z.D. designed and supervised the study, P.W. performed the experiments, Y.W., Y.C., F.Z., Y.L. and J.Z. enrolled patients, Z.H. and X.D. collected samples, P.W. and X.D. analyzed the data, P.W. wrote the manuscript, P.W., Y.Q. and X.D. revised the manuscript.

Acknowledgment: This work was funded by the Shanghai Benemae Pharmaceutical Corporation (Shanghai, China).

Disclosure: no potential conflict of interest relevant to this article was reported.

\section{REFERENCES}

1. Gallagher D, Heymsfield SB, Heo M, Jebb SA, Murgatroyd PR, Sakamoto $Y$. Healthy percentage body fat ranges: an approach for developing guidelines based on body mass index. Am J Clin Nutr. 2000;72(3):694-701.

2. He Y, Pan A, Wang Y, Yang Y, Xu J, Zhang Y, et al. Prevalence of overweight and obesity in 15.8 million men aged $15-49$ years in rural China from 2010 to 2014. Sci Rep. 2017;7(1):5012.

3. Zhai F, Wang H, Du S, HeY, Wang Z, Ge K, et al. Prospective study on nutrition transition in China. Nutr Rev. 2009;67 Suppl 1:S56-61.

4. Emerging Risk Factors Collaboration, Wormser D, Kaptoge S, Di Angelantonio E, Wood AM, Pennells L, Thompson A, et al. Separate and combined associations of body-mass index and abdominal adiposity with cardiovascular disease: collaborative analysis of 58 prospective studies. Lancet. 2011;377(9771):1085-95.

5. Prospective Studies Collaboration, Whitlock G, Lewington S, Sherliker P, Clarke R, Emberson J, Halsey J, et al. Body-mass index and cause-specific mortality in 900000 adults: collaborative analyses of 57 prospective studies. Lancet. 2009;373(9669):1083-96.

6. Ezzati M, Lopez AD, Rodgers A, Vander Hoorn S, Murray CJL. Selected major risk factors and global and regional burden of disease. Lancet. 2002;360(9343):1347-60.

7. Stevens GA, Singh GM, LuY, Danaei G, Lin JK, Finucane MM, et al. Global Burden of Metabolic Risk Factors of Chronic Diseases Collaborating Group (Body Mass Index). National, regional, and global trends in adult overweight and obesity prevalences. Popul Health Metr. 2012;10(1):22.

8. Wang C, Li J, Xue H, Li Y, Huang J, Mai J, et al. Type 2 diabetes mellitus incidence in Chinese: Contributions of overweight and obesity. Diabetes Res Clin Pract. 2015;107(3):424-32.

9. Andersen A, Lund A, Knop FK, Vilsbøll T. Glucagon-like peptide 1 in health and disease. Nat Rev Endocrinol. 2018;14(7):390-403.

10. Zhong X, Chen Z, Chen Q, Zhao W, Chen Z. Novel Site-Specific Fatty Chain-Modified GLP-1 Receptor Agonist with Potent Antidiabetic Effects. Molecules. 2019;24(4).

11. Baggio LL, Drucker DJ. Glucagon-like peptide-1 receptors in the brain: controlling food intake and body weight. J Clin Invest. 2014;124(10):4223-6.

12. Tao L, Wang L, Yang $X$, Jiang $X$, Hua F. Recombinant human glucagon-like peptide-1 protects against chronic intermittent hypoxia by improving myocardial energy metabolism and mitochondrial biogenesis. Mol Cell Endocrinol. 2019;481:95-103.

13. Enomoto $M$, Adachi $H$, Fukami $A$, Kumagai $E$, Nakamura $S$, NoharaY, et al. A Useful Tool As a Medical Checkup in a General Population-Bioelectrical Impedance Analysis. Front Cardiovasc Med. 2017;4:3-3.

14. Zhang $Y L$, Zhou $C$, Li XF, Yang MN, Tao L, Zheng $X Y$, et al. Beinaglutide showed significant weight-loss benefit and effective glycaemic control for the treatment of type 2 diabetes in a real-world setting: a 3-month, multicentre, observational, retrospective, open-label study. Obes Sci Pract. 2019 Jun 17;5(4):366-75.

15. Lorenz M, Lawson F, Owens D, Raccah D, Roy-Duval C, Lehmann $A$, et al. Differential effects of glucagon-like peptide-1 receptor agonists on heart rate. Cardiovasc Diabetol. 2017;16(1):6.

16. Shah M, Vella A. Effects of GLP-1 on appetite and weight. Rev Endocr Metab Disord. 2014 Sep;15(3):181-7.

17. VilsbølIT, Christensen M, Junker AE, Knop FK, Gluud LL. Effects of glucagon-like peptide-1 receptor agonists on weight loss: systematic review and meta-analyses of randomised controlled trials. BMJ. 2012;344:d7771.

18. Neeland IJ, Ross R, Despres JP, MatsuzawaY,Yamashita S, Shai I, et al.; International Atherosclerosis Society; International Chair on Cardiometabolic Risk Working Group on Visceral Obesity. Visceral and ectopic fat, atherosclerosis, and cardiometabolic disease: a position statement. Lancet Diabetes Endocrinol. 2019;7(9):715-25.

19. Wajchenberg BL. Subcutaneous and visceral adipose tissue: Their relation to the metabolic syndrome. Endocr Rev. 2000;21(6):697-738.

20. Kaur P, Reis MD, Couchman GR, Forjuoh SN, Greene JF, Asea A. SERPINE 1 Links Obesity and Diabetes: A Pilot Study. J Proteomics Bioinform. 2010 Jun 1;3(6):191-9.

21. Gaspari T, Liu HB, Welungoda I, Hu YS, Widdop RE, Knudsen LB, et al. A GLP-1 receptor agonist liraglutide inhibits endothelial cell dysfunction and vascular adhesion molecule expression in an ApoE-/- mouse model. Diab Vasc Dis Res. 2011;8(2):117-24.

22. Liu $H B$, Dear $A E$, Knudsen LB, Simpson RW. A long-acting glucagon-like peptide-1 analogue attenuates induction of plasminogen activator inhibitor type-1 and vascular adhesion molecules. J Endocrinol. 2009;201(1):59-66.

23. Kamada $Y$, Takehara T, Hayashi N. Adipocytokines and liver disease. J Gastroenterol. 2008;43(11):811-22.

24. Chaudhuri A, Ghanim H, Vora M, Sia CL, Korzeniewski K, Dhindsa $\mathrm{S}$, et al. Exenatide exerts a potent antiinflammatory effect. J Clin Endocrinol Metab. 2012;97(1):198-207.

25. Hogan AE, Gaoatswe G, Lynch L, Corrigan MA, Woods C, O'Connell $\mathrm{J}$, et al. Glucagon-like peptide 1 analogue therapy directly modulates innate immune-mediated inflammation in individuals with type 2 diabetes mellitus. Diabetologia. 2014;57(4):781-4.

26. Pastel E, McCulloch LJ, Ward R, Joshi S, Gooding KM, Shore AC, et al. GLP-1 analogue-induced weight loss does not improve obesityinduced AT dysfunction. Clin Sci (Lond). 2017;131(5):343-53.

27. Considine RV, Sinha MK, Heiman ML, Kriauciunas A, Stephens TW, Nyce MR, et al. Serum immunoreactive-leptin concentrations in normal-weight and obese humans. N Engl $\mathrm{J}$ Med. 1996;334(5):292-5.

28. Al Maskari MY, Alnaqdy AA. Correlation between Serum Leptin Levels, Body Mass Index and Obesity in Omanis. Sultan Qaboos Univ Med J. 2006;6(2):27-31.

29. Selvin E, Paynter NP, Erlinger TP. The Effect of Weight Loss on C-Reactive Protein: A Systematic Review. Arch Intern Med. 2007;167(1):31-9.

30. Mazidi M, Karimi E, Rezaie P, Ferns GA. Treatment with GLP1 receptor agonists reduce serum CRP concentrations in patients with type 2 diabetes mellitus: A systematic review and meta-analysis of randomized controlled trials. J Diabetes Complications. 2017;31(7):1237-42. 